\title{
A COMPARATIVE STUDY OF DIMENSION REDUCTION METHODS COMBINED WITH WAVELET TRANSFORM APPLIED TO THE CLASSIFICATION OF MAMMOGRAPHIC IMAGES
}

\author{
N. Hamdi ${ }^{1}$, K. Auhmani ${ }^{12} *$, M. M. Hassani ${ }^{1}$ \\ ${ }^{1}$ Equipe I2SP, Département de Physique \\ Faculté des sciences Semlalia, \\ Université Cadi Ayyad, \\ Marrakech 40000 Maroc \\ ${ }^{2}$ Ecole Nationale des sciences appliquées \\ Université Cadi Ayyad \\ BP 63 Safi 46000, Maroc,
}

\begin{abstract}
In this paper, we present a comparative study of dimension reduction methods combined with wavelet transform. This study is carried out for mammographic image classification. It is performed in three stages: extraction of features characterizing the tissue areas then a dimension reduction was achieved by four different methods of discrimination and finally the classification phase was carried. We have late compared the performance of two classifiers KNN and decision tree.

Results show the classification accuracy in some cases has reached $100 \%$. We also found that generally the classification accuracy increases with the dimension but stabilizes after a certain value which is approximately $d=60$.
\end{abstract}

\section{KEYWORDS}

Dimension reduction, Classification, Feature extraction, Mammographic images.

\section{INTRODUCTION}

The problem of feature selection in classification often arises when it comes to consider a very large number of variables. In recent years the need has evolved with the manipulation of very large databases and especially in areas such as genetic field and image processing field [1]. Hence the need to reduce the number of variables (features) exploited for classifying an image or an object.

Dimension reduction algorithms used for this purpose are therefore trying to find a projection of the data in a space of reduced dimension, while preserving the information contained therein. This projection may be linear or nonlinear.

Our goal in this paper is to perform a comparative study of the effect of dimension reduction methods on the classification accuracies of breast cancer (mammographic image). We will 
present the performance of the following methods: locality-preserving projection (LPP), locally linear embedding (LLE), Isometric Mapping (Isomap) and spectral regression (SR). We will present the results of the classification versus the reduced space dimension, the method used for the reduction and the type of the wavelet transform used for the extraction of features.

The classification task of mammographic image has been carried out through different stages. The first step is to prepare the vector containing the variables describing the image. The second phase aims to reduce the size (dimension) of this vector. The last step is to find the class of the incoming picture. This last step begins with a learning phase of the classifier and then returns the final result.

\section{MATERIALS AND METHODS}

\subsection{Features generation}

Initially, the features are calculated to form the feature vector for subsequent learning step. These features were calculated on a set of two classes labeled images (normal and abnormal). These images are firstly preprocessed and transformed into the frequency domain by three types of wavelet transforms.

The following features are extracted:

- A vector of 24 texture descriptors is formed from a multi-level histogram of 3, 5, 7, and 9 bins [2].

- A vector of descriptors is calculated from the first order of statistical moments which are based on one-dimensional distribution of the gray levels of an image. This distribution can be described by the moments of order 1 to 4: Mean, Variance, and Fisher coefficients, Skewness and Kurtosis. However, such indicators do not take into account the spatial dependencies, which are inseparable from the concept of texture. They are therefore not sufficient to fully characterize the texture and are often associated with dynamic statistical descriptors [3]. We calculate the mean, the variance, the Skewness and the kurtosis of the image, in four different directions $(0,45,90$ and 135 degrees). Then an histogram is calculated for each of the three bins resulting series, which gives 4 $* 4 * 3=48$ descriptors.

- Three of the six parameters introduced by Tamura are used to characterize textures by Tamura method, namely, coarseness, contrast and directionality. We also calculate an histogram of 3 bins on the coarseness. In total, this group of descriptors forms a vector of six texture features $[4,5]$.

- Radon's characteristics are calculated for angles $0^{\circ}, 45^{\circ}, 90^{\circ}$ and $135^{\circ}$. We also calculate the histogram of 3 bins for the four series, which gives a vector of 12 features $[6,7]$.

- Zernike's moments of order $n=12$ are calculated corresponding to 49 features [8].

The extracted features are shown in Table 1.

In order to exploit the advantage of each feature, we merge them into a single feature vector. Depending on the used wavelet transform, there will be a different number of features. If the used wavelet transform is the double density discrete wavelet transform (3DWT), the image is divided into nine sub-images, so the number of features is $9 * 139=1251$. If the transformation is based on the stationary wavelet transform (SWT) or the discrete wavelet transform (DWT), the original image is decomposed into four sub-images, and the number of calculated features is $4 * 139=556$. Each original image will be represented by a group of 556 or 1251 genes. Our original database consisted of 107 patients divided into two classe: 56 tumoral samples and 51 normal samples. 
Table 1: Description of calculated features

\begin{tabular}{|c|c|}
\hline Feature type & Number \\
\hline Zernike's Moment & 49 \\
Radon's Transform & 12 \\
Multi-scale Histogram & 24 \\
Tamura's features & 6 \\
The 4 Moments & 48 \\
\hline
\end{tabular}

\subsection{Dimension reduction}

The number of features appears to be very large, which may affect the results of the classification. For this reason, we thought to make a reduction of the number of features to be used by eliminating redundant and irrelevant ones.

We applied four approaches of discriminant analysis, introduced above, on the feature vector to extract a sub-optimal space improving learning and classification. We examine the effectiveness of the reduced space in relation with the considered classification techniques. We will also perform a comparison between the wavelet transforms in order to form the final combination of best compromise: best wavelet transform / best reduced space.

\subsection{Benchmark}

Our benchmark (Figure 1) is divided in four parts. First, the original mammographic image will undergo pre-processing to reduce noise, enhance the features and enhance the presentation. The next part deals with the extraction of features as described above. In the third part, the studied dimension reduction methods will be applied to the complete dataset. The low-dimensional datasets will then be classified by two different classifiers, namely KNN and decision tree. To evaluate and compare the performance of each method, the classification accuracies of KNN on decision tree will be presented versus reduced dimension and wavelet transform type.

\subsection{Dataset}

The methods were tested on the original mammographic images of MIAS database [9]. These images of original size $1024 * 1024$ are accompanied by the opinion of the radiologists that specify the class of the image, the type of microcalcifications, the center of clusters and other informations. We decomposed the images into regions of interest (ROI) of size $256 * 256$ and applied our benchmark on the ROI. 


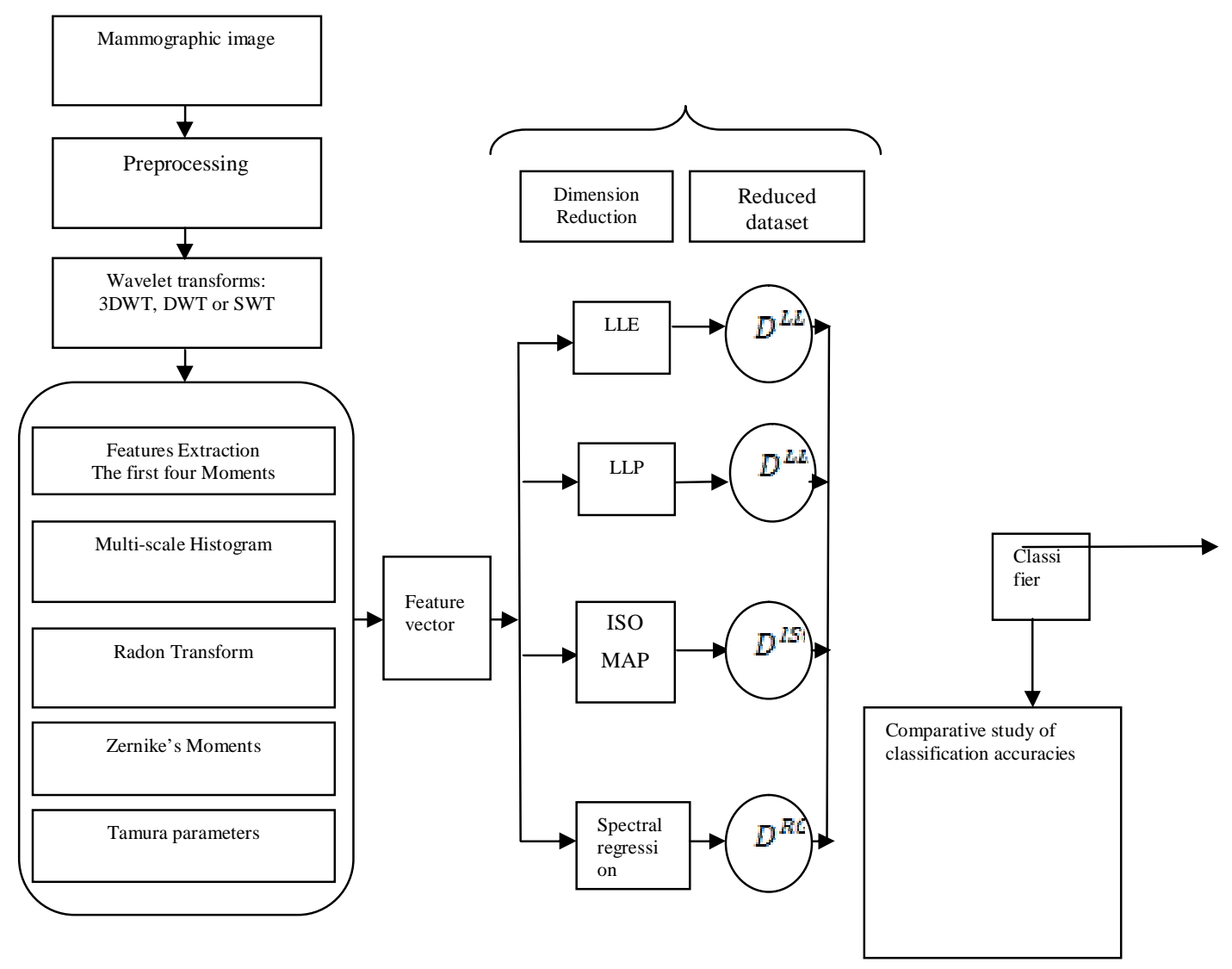

Figure 1 Benchmark of the studied methods

\section{RESULTS AND DISCUSSION}

\subsection{Dimension influence}

The following sections present the results of breast cancer classification. In Figure 2 we present the classification accuracies versus dimension $\mathrm{d}$ and dimension reduction method. We can easily deduce that accuracy is significantly better if features are extracted from an image that has undergone a wavelet transform than if the features are calculated directly on the original image. Indeed in Figure 2.a, the average of classification accuracy is about $60 \%$ and the maximum value of this rate is $95.32 \%$, while in Figure 2.b the average is $95 \%$, while the maximum value is $100 \%$. It is clear that the distinction offered by the wavelet transform, namely its ability to represent the texture at various scales, is an advantage. From these results, we decide for our later study to apply a wavelet transform to mammographic images before extracting features. 
International Journal of Computer Science \& Information Technology (IJCSIT) Vol 6, No 6, December 2014

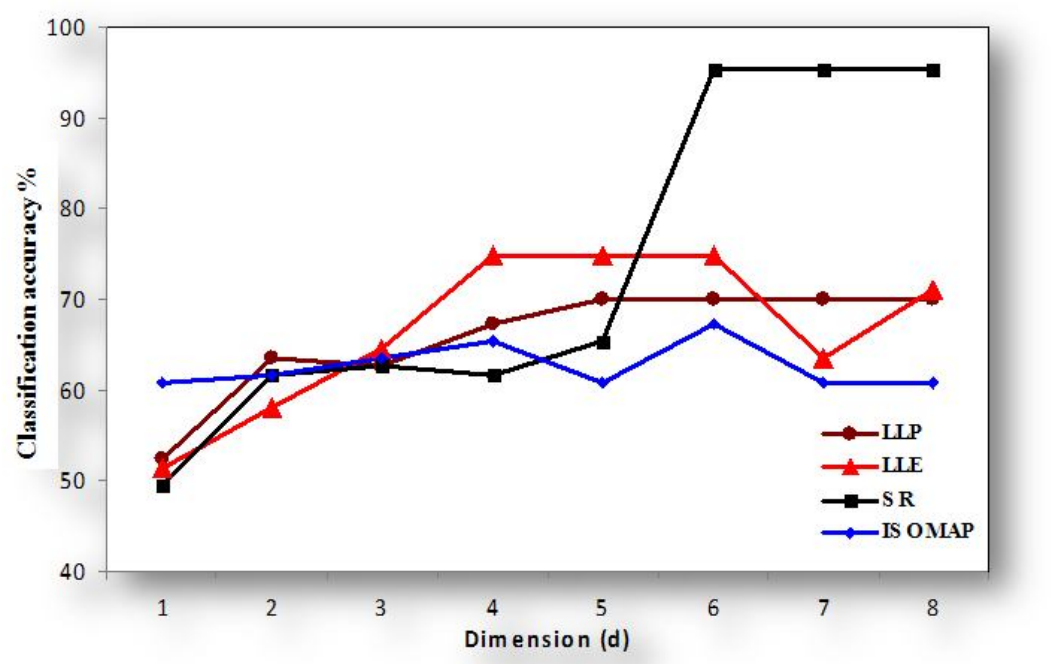

(a)

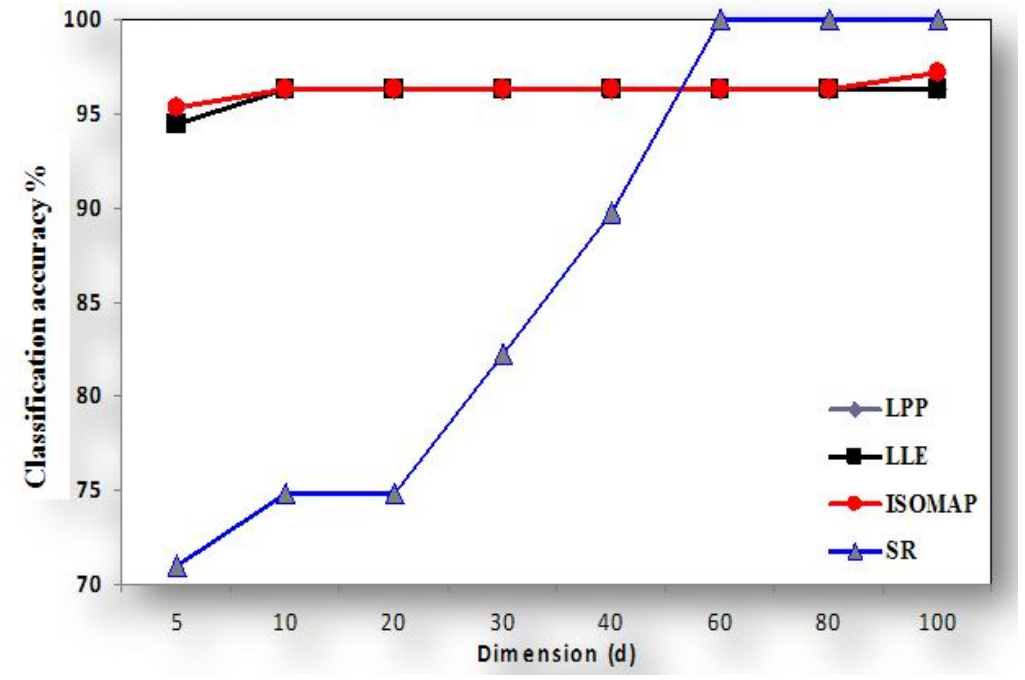

(b)

Figure 2: Effect of different dimension reduction methods on classification accuracy; (a) features extracted from original image, (b) features extracted from transformed image by the double-density discrete wavelet transform 3DWT.

We observe an interesting general result: the classification accuracy increases with the reduced space dimension. Figures 2, 3 and 4 show the effect on classification performance for different wavelet transforms and different methods of dimension reduction.

Figure 2.b presents the classification accuracy versus space dimension (d). Features are extracted from image transformed by the double-density wavelet transform 3DWT. We remark that the best performance is $100 \%$ (all the 107 samples are correctly classified) obtained with Spectral regression method (SR) for space dimension $\mathrm{d}=60$. However, the comparison of the accuracies corresponding to LLP, LLE and Isomap, shows that reducing the space for small size $(d=5)$ the classification accuracy of $95.3 \%$ is held.

Figures 3 and 4 show the accuracy versus dimension d, features are extracted from mammographic images transformed by discrete wavelet transform (DWT) and stationary wavelet 
transform (SWT) respectively. The classification accuracy is less performant than the results corresponding to the 3DWT. The accuracy reaches $96,2 \%$ and $97,2 \%$ for DWT and SWT respectively in a reduced space with SR method.

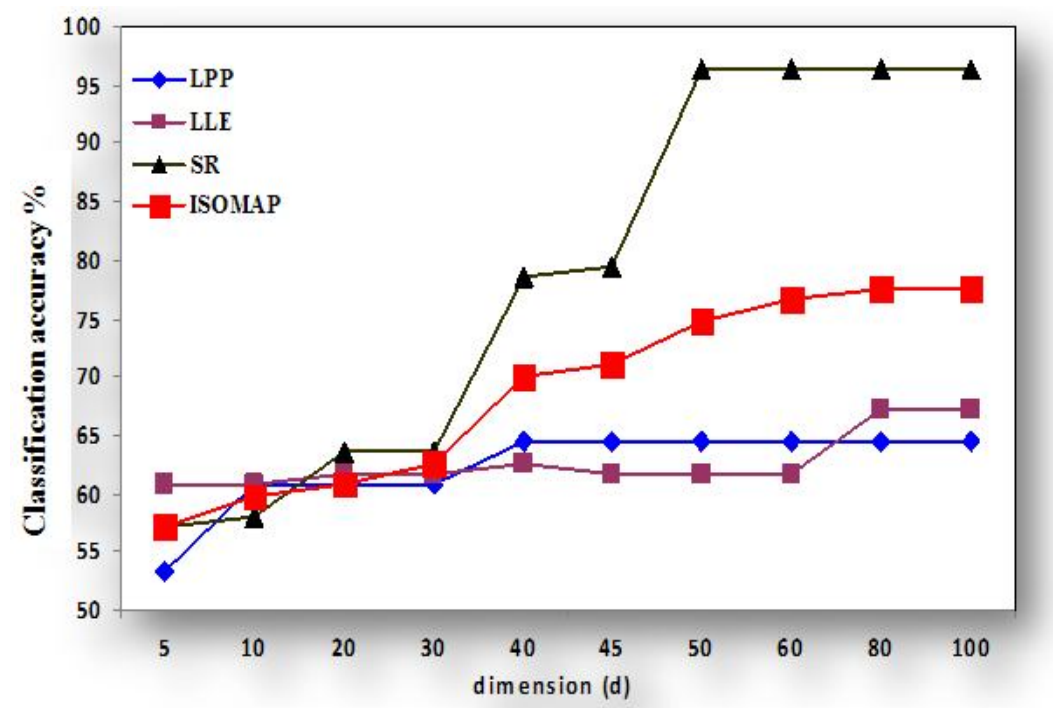

Figure 3: Effect of different dimension reduction methods on classification accuracy, features extracted from transformed image by the discrete wavelet transform DWT

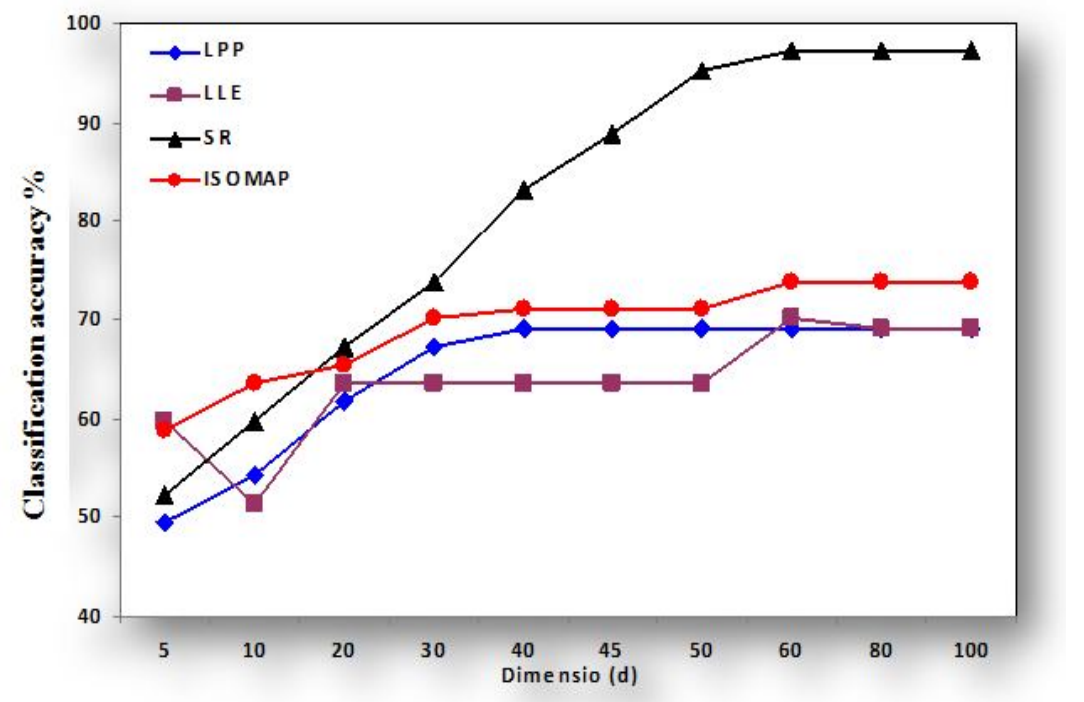

Figure 4 Effect of different dimension reduction methods on classification accuracy: features extracted from transformed image by the stationary wavelet transform SWT.

Figures show clearly that for small dimensions, i.e. $\mathrm{d}=10,20,25$, the accuracy with SR method increases with dimension up stabilization for $\mathrm{d}=60$. This means that classification task is easier with high dimensions than low dimensions. Moreover, we find this behaviour for the other methods: classification accuracy increases slightly as the data size increases. 


\subsection{Performances versus classifier type}

After the evaluation of dimension reduction impact on the classification accuracy, the optimization of our system must take into account the influence of the classifier type. In order to measure this impact, a classification was performed by using extracted features from image transformed by DWT technique. Two different classifiers are studied: decision tree and k-nearest neighbours $(\mathrm{KNN})$. The algorithm of k-nearest neighbours was executed for $\mathrm{k}=1$. Figure 5 shows that the classification with decision tree classifier improves the recognition rate by $1.5 \%$ compared to that obtained with KNN. We also observe that the two classifiers gives the same performance after a certain dimension threshold (here $\mathrm{d}=50$ ). The dimension reduction method used for this comparison is the SR one.

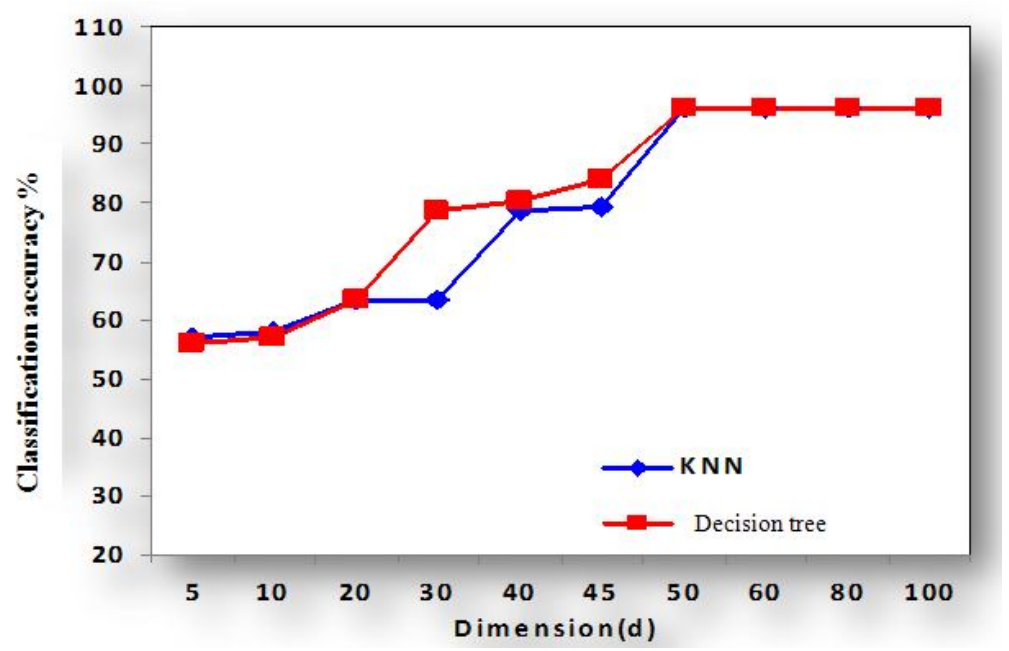

Figure 5 Comparison of classification accuracy for two classifiers, features extracted from image transformed by the DWT and dimension reduction considered here is SR.

\subsection{Interpretation}

All the four algorithms (of dimension reduction) that we have used in the experiment try to optimize an objective function. The spectral regression method performs a discriminant analysis of variables maximizing inter-class dispersion (dispersion of cluster centers) and minimizing intra-class dispersion (dispersion in a class about its center), whereas the other methods depend on the number of $\mathrm{k}$ nearest neighbours used to construct the graph.

Spectral regression method achieves better classification than the other methods, which verifies the effectiveness of regulation for classification [10]. This is due to the fact that it structures data to make different classes disjoint. However, this transformation associated with a classification model that operates its own data processing (eg SVM) can cause a problem of over-learning and decreased performance.

The presented results allow us to determine the optimal dimensions as a compromise between the size of the feature vector and the classification performance: from the 1251 features the spectral regression retains an average of 60 features to describe each class. 
International Journal of Computer Science \& Information Technology (IJCSIT) Vol 6, No 6, December 2014

\section{CONCLUSION}

The dimensionality reduction algorithms attempt to find a projection of the data in a space of smaller dimension, while preserving the information contained in those. In this paper, we presented a process of tissue classification applied to the evaluation of the pathologic state of breasts. This process was performed in three stages: extraction of features characterizing the tissue areas then a dimension reduction was achieved by four different methods of discrimination and finally the classification phase was carried out.

During the experimental phase, we compared the different techniques of dimension reduction associated with a wavelet transform applied to the image before the features extraction process. We have late compared the performance of two classifiers KNN and decision tree.

We have reached a classification accuracy of $100 \%$ for some combinations. We also found that generally the classification accuracy increases with the dimension but stabilizes after a certain value which is $d=60$.

\section{REFERENCES}

[1] Ali El Akadi, «Contribution à la sélection de variables pertinentes en classification supervisée: application à la sélection des gènes pour les puces à ADN et des caractéristiques faciales »; these de doctorat, Faculté des sciences Rabat, 2012.

[2] Hadjidemitriou, E., Grossberg, M. D., \& Nayar, S. K.. Multiresolution histograms an their use for recognition. IEEE Transactions on Pattern Analysis and Machine Intelligence, 26 (7), 831-847. (2004)

[3] S. HERLIDOU, Caractérisation tissulaire en IRM par l'analyse de texture. Étude du tissu musculaire et de tumeurs intracrâniennes, université de Renne1, 1999.

[4] H. TAMURA, S. MORI et T. YAMAWAKI. Texture features corresponding to visual perception IEEE Transactions on Systems, Man and Cybernetics, SMC-8(6):460-473, 1978.

[5] P. HOWARTH et S. RÜGER. Evaluation of texture features for content-based image retrieval. In Proceedings of the International Conference on Image and Video Retrieval (CIVR'04), volume LNCS 3115, pages 326-334, Dublin, Ireland, jul 2004.

[6] Deans, S.R. Hough Transform from the Radon Transform_, IEEE Trans. On Patt. Anal. and Mach. Intell., Vol. PAMI-3, No. 2, pp. 185_188, 1981.

[8] Murphy, L.M. Linear feature detection and enhancement in noisy images via the Radon transform, Pattern Recognition Letters, No. 4, pp. 279_284, 1986.

[9] C.-W. Chong, P. Raveendran, and R. Mukun-dan. A comparative analysis of algorithms for fast computation of zernike moment. Pattern Recognition, 36:731-742, 2003.

[10] http://www.wiau.man.ac.uk/services/mias/miasweb.html

[11] T. Hastie, A. Buja, and R. Tibshirani. Penalized discriminant analysis. Annals of Statistics, 23:73$102,1995$. 